\title{
Human Shape Tracking for Gait Recognition Using Active Contours with Mean Shift
}

\author{
Kyung Su Kwon ${ }^{1}$, Se Hyun Park ${ }^{2}$, Eun Yi Kim ${ }^{3}$, and Hang Joon Kim ${ }^{1}$ \\ ${ }^{1}$ Department of Computer Engineering, Kyungpook National Univ., Korea \\ \{kskwon, hjkim\}@ailab.knu.ac.kr \\ ${ }^{2}$ School of Computer and Communication, Daegu Univ., Korea \\ sehyun@daegu.ac.kr \\ ${ }^{3}$ Department of Internet and Multimedia Engineering, Konkuk Univ., Korea \\ eykim@konkuk.ac.kr
}

\begin{abstract}
In this paper, we present a human shape extraction and tracking for gait recognition using geodesic active contour models(GACMs) combined with mean-shift algorithm. The active contour models (ACMs) are very effective to deal with the non-rigid object because of its elastic property, but they have the limitation that their performance is mainly dependent on the initial curve. To overcome this problem, we combine the mean-shift algorithm with the traditional GACMs. The main idea is very simple. Before evolving using levelset method, the initial curve in each frame is re-localized near the human region and is resized enough to include the targe object. This mechanism allows for reducing the number of iterations and for handling the large object motion. Our system is composed of human region detection and human shape tracking. In the human region detection module, the silhouette of a walking person is extracted by background subtraction and morphologic operation. Then human shape are correctly obtained by the GACMs with mean-shift algorithm. To evaluate the effectiveness of the proposed method, it is applied the common gait data, then the results show that the proposed method is extracted and tracked efficiently accurate shape for gait recognition.
\end{abstract}

Keywords: Human Shape Tracking, Geodesic Active Contour Models, Mean Shift, Gait Recognition.

\section{Introduction}

During the decades, the gait recognition has recently has received many attentions from computer vision researchers [1-5]. The human gait recognition is the process of identifying individuals by way of their walking at a distance [1]. It has many applications such as visual surveillance, security management, smart interface, and the human identification. The major issue related to the gait recognition is to generate the accurate human shapes. Generally, the human gait is determined by their weight, limb length, habitual posture and so on [2]. To use these features in gait recognition, the technique should be firstly developed to extract and track the human shape. 
However, it is difficult problem as the human shape is deformed variously in an image sequence and also includes discontinuous and dim edge [6].

Recently, active contour models (ACMs) have been increasingly used in object extraction and tracking. ACMs are effective to describe the elastic properties of the non-rigid objects, so that can provide the detailed analysis of the shape deformation during the human is moving through the whole video sequences.

In this paper, we present a human shape extraction and tracking for gait recognition using geodesic active contour models (GACMs) combined with mean-shift algorithm. The GACMs are specially designed to overcome the major drawback of the ACMs that the performance is higly dependent on the initial curve [7]. The main idea is very simple. Before evolving using level-set method, the initial curve in each frame is relocalized near the human region and is resized enough to include the targe object.

Fig. 1 shows the overview of the proposed system, which is composed of human region detection and human shape tracking. In the human region detection, the silhouette of a walking person is extracted by background subtraction and morphologic operation. Thereafter, in the human shape tracking, the human shape is correctly obtained by the GACMs with mean-shift algorithm. The tracking is performed by two steps: curve localization step and curve deformation step. When given the initial curve of the current frame from the object contour of the previous frame, the initial curve is firstly localized near the target object using mean-shift algorithm, and then it is deformed using a level-set method.

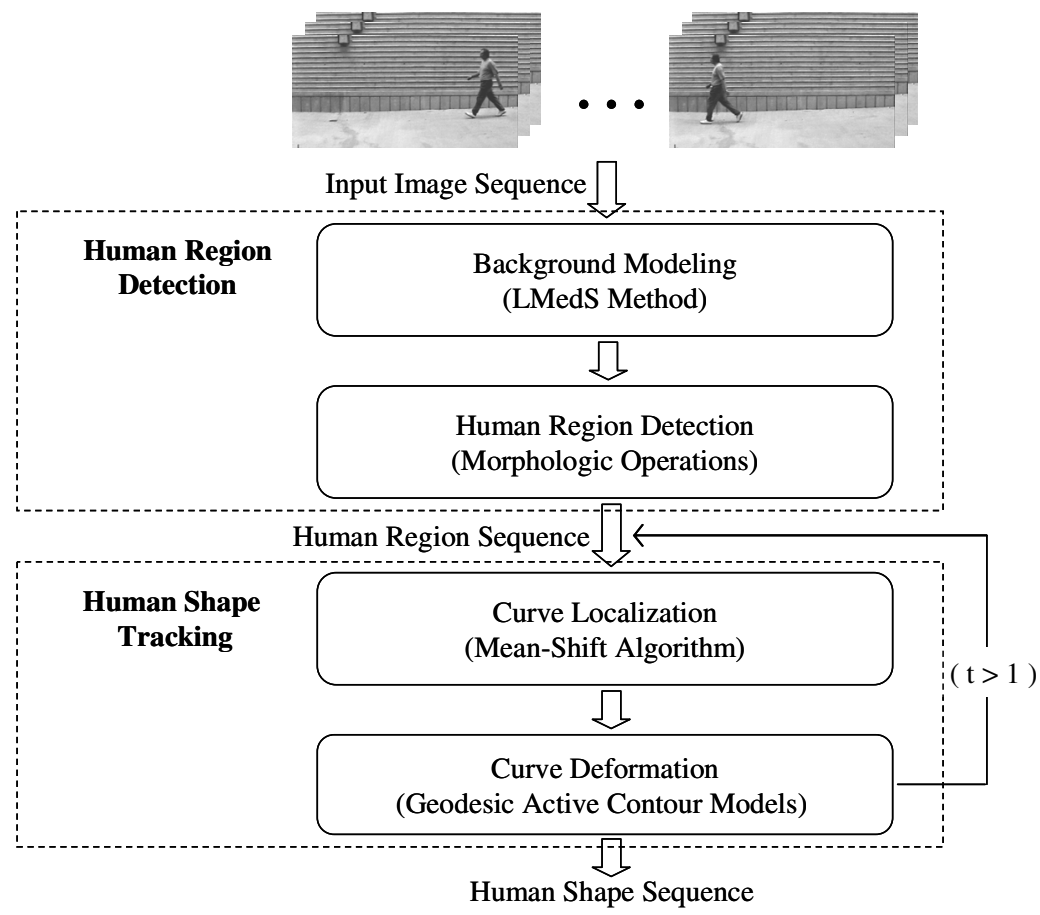

Fig. 1. Overview of the proposed method 
To evaluate the effectiveness of the proposed method, it is applied the common gait data [8] which include a walking person. Experiment results show that the proposed method is extracted and tracked efficiently accurate shape for gait recognition.

The remainder of this paper is organized as follows. Section 2 describes the human region detection. Section 3 describes the human shape extraction and tracking. Experimental results are presented in Section 4. Finally, the conclusion is drawn in Section 5 .

\section{Human Region Detection}

In this section, we firstly present the human region detection algorithm for getting shape information of a walking person from each frame of an image sequence. This is the preprocessing phase of human tracking. And it is composed two steps: background modeling and human region detection.

\subsection{Background Modeling}

To extract the human region, background subtraction based change detection in between the current image and background image is adopted. We have assumed that the camera and background is static, and the only moving object in image sequences is a walking person. The background image is modeled by the Least Median of Squares (LMedS) method [9] because, the used gait data [8] is not included a background image. Using this method, background can be modeled from a sequence of including moving objects. Let $I$ represent a sequence including $N$ images. We can get the reliable background through $N$ over 70 . The resulting background $B(i, j)$ can be computed by

$$
B(i, j)=\min _{q} \operatorname{med}_{t}\left(I_{i j}^{t}-q\right)^{2},
$$

where $q$ is the background intensity value to be determined for the pixel location $(i, j)$ on image coordinate, med represents the median value, and $t$ represents the frame index within $1-N$.

\subsection{Foreground Region Detection}

After the background modeling, each foreground region is detected by subtraction between current image and background image. In our test data, the human shadow is existed with a horizontal slant. The human shadow is removed by convolution of vertical Sobel mask. The detected edge image is then binarized by Ostu method. Accurate human region is detected by morphologic closing operation and removal of inner black points [6]. Fig. 2 shows the process of human region detection. Thereafter, we can get the rough foreground region with cracks and dim edges without human shadow. After projection on horizontal and vertical direction for the foreground region, gained range of region projection is used to determine initial curve's position (the centroid) and size of GACMs. 


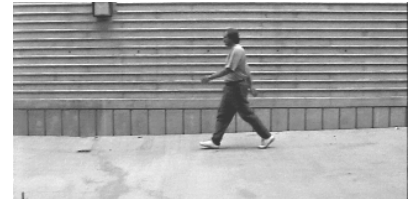

(a)

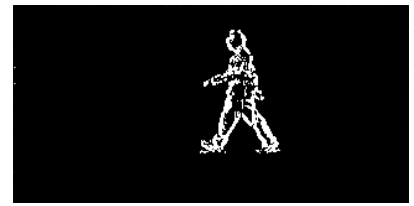

(c)

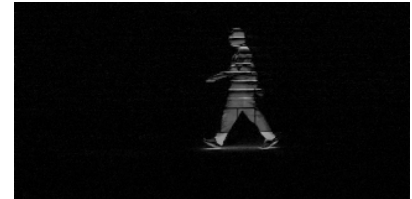

(b)

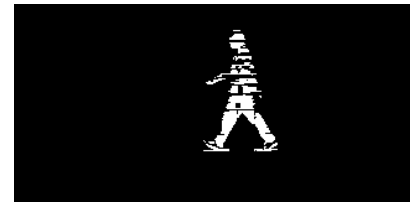

(d)

Fig. 2. The procedure of human region detection: (a) an original image, (b) the result of background subtraction, (c) an edge image after performing the vertical Sobel operation, (d) a foreground image without human shadow

\section{Human Shape Tracking}

After human region is detected, GACMs combined with a mean-shift algorithm for human shape tracking is used. In general, the ACMs are very effective to extract the boundary of the non-rigid object. Howere, they have the limitation that their performance is mainly dependent on conditions of the initial curve such as location and size. To solve this problem, we present a method of human shape tracking using a GACM and a mean-shift algorithm. In this method, the tracking process is achieved by two steps: curve localization step and curve deformation step. In first step, a meanshift algorithm is used to move the initial curve near the human region. And the relocalized init curve is resized enough to include the target region. In the second set, the curve is deformed by a level-set method.

\subsection{Curve Localization Using Mean-Shift}

The mean-shift algorithm is a nonparametric technique that climbs the gradient of a probability distribution to find the nearest dominant mode [10]. This algorithm has recently been used as an efficient technique for object tracking [11]. In this paper, a mean-shift algorithm to replace the curve location in every frame excepted the first frame is used. And the curve location is determined by the number of pixels belong to human region in search window. The human region is represented by a binary foreground image $F(i, j)$.

The mean-shift algorithm iteratively replace the search window location (the centroid) until the moving distance of window below the threshold. The search window location is simply computed as follows $[12,13]$ :

$$
x=M_{10} / M_{00} \text { and } y=M_{01} / M_{00},
$$

where $M_{a b}$ is the $(a+b)$ th moment as defined by

$$
M_{a b}(W)=\sum_{i, j \in W} i^{a} j^{b} F(i, j) .
$$


The object location is obtained by successive computations of the search window location $(i, j)$. The center of search window $W$ is initialized with the center of the initial curve. And its size is updated in proportional to the amount of object's motion at each frame as follows:

$$
\begin{aligned}
& W_{\text {width }}=\max \left(\alpha\left(\left|m_{x}^{t}-m_{x}^{t-1}\right|-B_{\text {width }}\right), 0\right)+\beta B_{\text {widh }} \text { and } \\
& W_{\text {height }}=\max \left(\alpha\left(\left|m_{y}^{t}-m_{y}^{t-1}\right|-B_{\text {height }}\right), 0\right)+\beta B_{\text {height }},
\end{aligned}
$$

where $\alpha$ and $\beta$ is a constant, and the $t$ is the frame index. Fig. 3 shows the curve localization by mean-shift algorithm. After the initial curve is localized, to completely surround the target region, the initial curve firstly is resized to outward of human region.

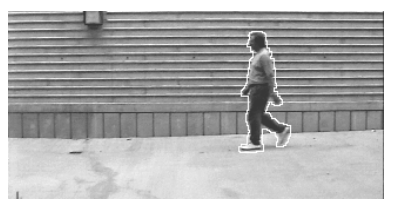

(a)

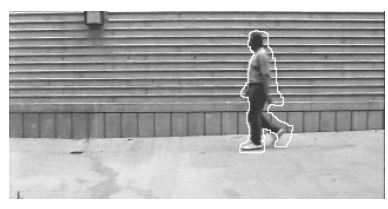

(b)

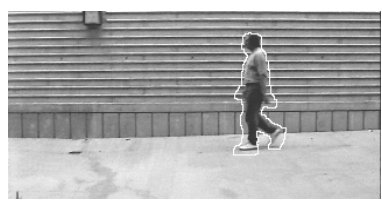

(c)

Fig. 3. Curve localization: (a) the result of curve evolving in the first frame, (b) the init curve in the second frame, and (c) the result of curve re-localization in the second frame

\subsection{Curve Deformation Using Level-Set}

Then the re-localized and scaled curve is deformed until it matches the human boundary using GACs. The GACMs was proposed by Vicent Caselles as a geometric alternative for snakes with the object of finding the curve $C(q)$ that minimizes the following energy [12]:

$$
E(C)=\int_{0}^{1} \mathrm{~g}\left(|\nabla I(C(q) \mid)| C^{\prime}(q) \mid d q,\right.
$$

where $C^{\prime}(q)$ is the partial derivative of curve, $q$ is its parameter and $g($.$) is a$ monotonically decreasing function such as a Gaussian and delta function.

The object boundary detection is to finding the curve that best take into account image characteristics. In order to minimize the energy $E$, the steepest-descent method, Euler-Lagrange equation is used. According to it, the curve evolution equation is derived as following:

$$
C_{t}=g(|\nabla I|) k \vec{N}-(\nabla g(|\nabla I|) \vec{N}) \vec{N},
$$

where $k$ is the Euclidean curvature, $\vec{N}$ is the unit inward normal vector and $t$ denotes the time as the contour evolves.

The geodesic curve equation (5) was implemented using level-set technique. We represent curve $C$ implicitly by the zero level-set of function $u: \Re^{2} \rightarrow \mathfrak{R}$, with the 
region inside $C$ corresponding to $u>0$. Accordingly, Eq. (5) can be rewritten by the following equation, which is a level-set evolution equation [7]:

$$
u_{t}=g(|\nabla I|) k|\nabla u|+\nabla g(|\nabla I|)|\nabla u|
$$

The unit inward normal vector $\vec{N}$ and the curvature value $k$ is estimated from the level-set function $u$ as following:

$$
\vec{N}=-\frac{\nabla u}{|\nabla u|}, \quad k=\operatorname{div}\left(\frac{\nabla u}{|\nabla u|}\right) .
$$
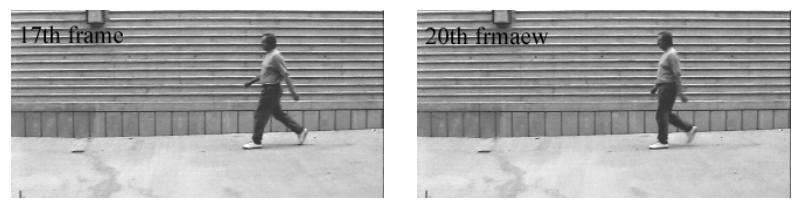

(a)
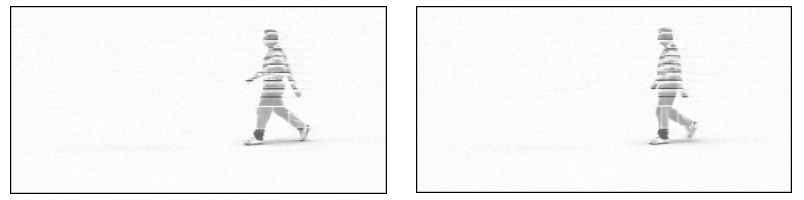

(b)
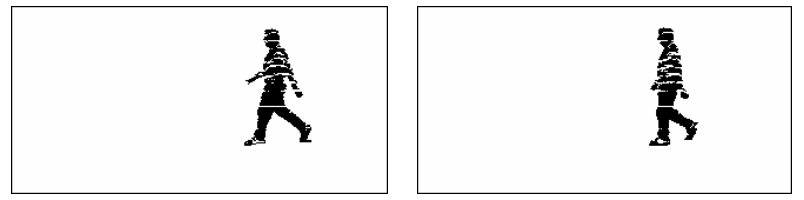

(c)
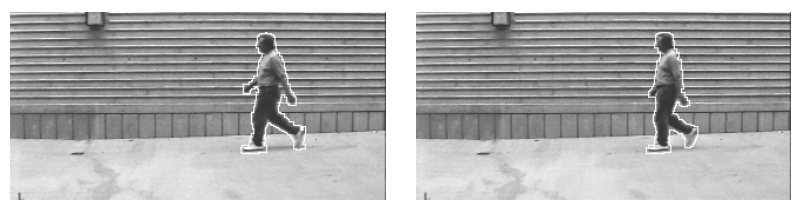

(d)
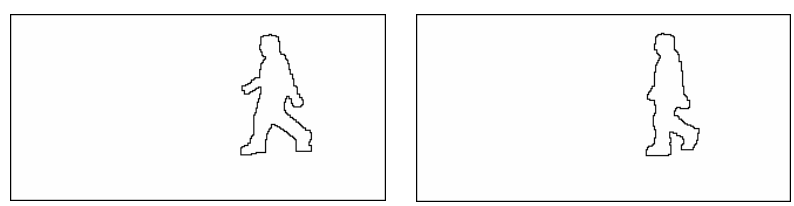

(e)
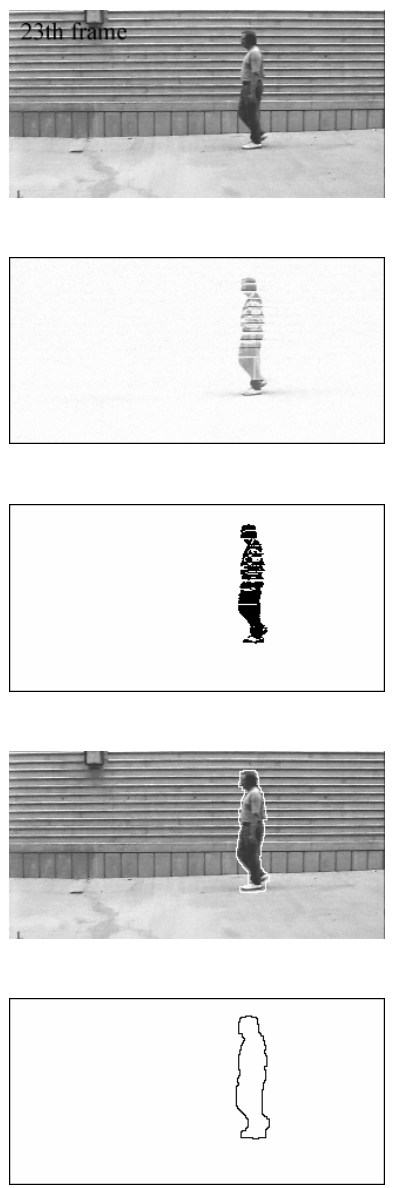
Fig. 4. Results of human region detection and shape tracking: (a) the input images, (b) the
background subtraction results, (c) the human region detection results, (d) the human shape tracking results, (e) the extracted human shape is shown 
To set up the initial level set values, we use a Euclidian distance mapping technique which is computed Euclidian distance between each pixel of the image and initial curve's centroid. And the evolving area is determined by narrow band approach, which is defined around the latest contour position and the level-set function is updated only within a set of narrow band pixels. The curve evolving is terminated when the difference of the number of pixel inside contour $C$ is less than a threshold value chosen manually.

\section{Experimental Results}

The proposed system was implemented using MS Visual C++ 6.0 and Intel's OpenCV beta3.1. The computer is a PentiumIV-2.8GHz with the Windows XP operation system. The proposed system was evaluated on the UCSD database [8]. The image size is a $320 \times 160$. It was taken in the outdoor and obtained at a distance from the camera. In the database, 6 persons were performed their walking 7 times. As such, 42 sequences were included in the UCSD database. For 42 sequences, the proposed method was evaluated. Then an example of the results is shown in Fig. 4. It shows that Fig.4 (a) is input images, Fig.4 (b) is background subtraction results, Fig.4 (c) is human region detection results based on morphologic operation, Fig.4 (d) is results of human shape tracking GACs with mean-shift algorithm, and Fig.4 (e) is shown the extracted human shape.

To fully demonstrate the effective of the proposed method, it was tested with various subjects, and then the results are shown in Fig. 6. As shown in Fig. 6, the proposed method yields accurate human shape tracking results, so that it can be used effectively to extract the gait features for human identification.

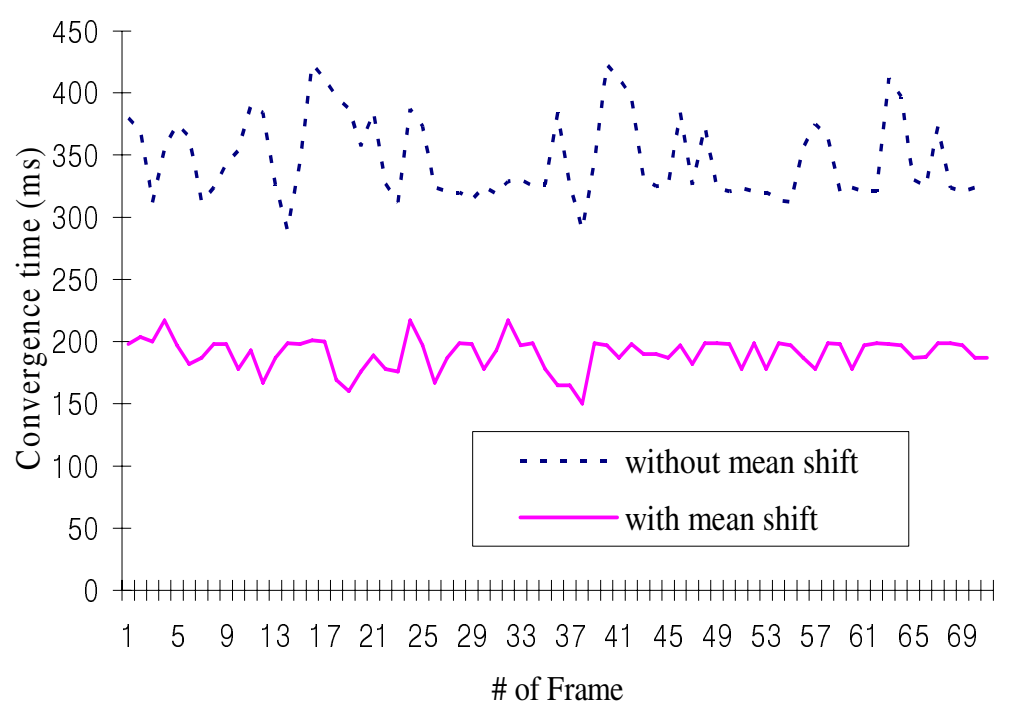

Fig. 5. Comparison of two methods in term of convergence time 

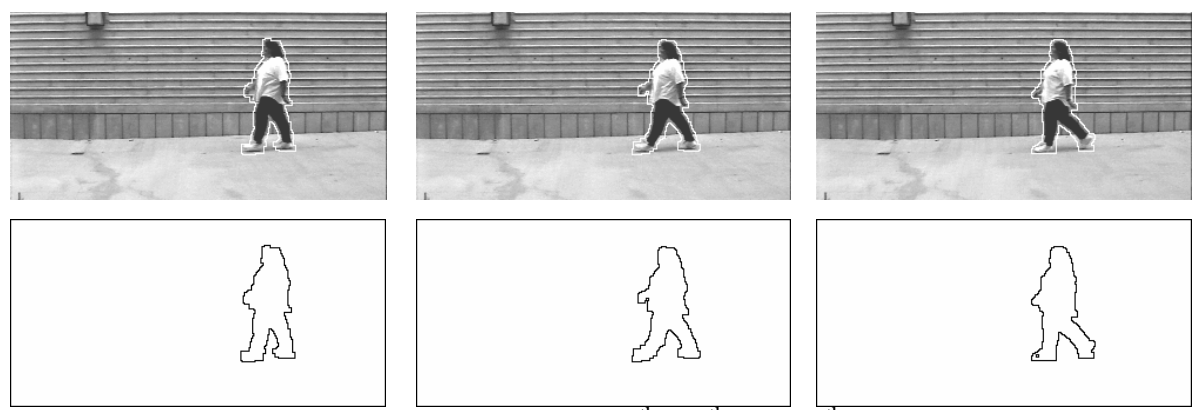

(a) the person $\# 2\left(25^{\text {th }}, 28^{\text {th }}\right.$ and $28^{\text {th }}$ frame $)$
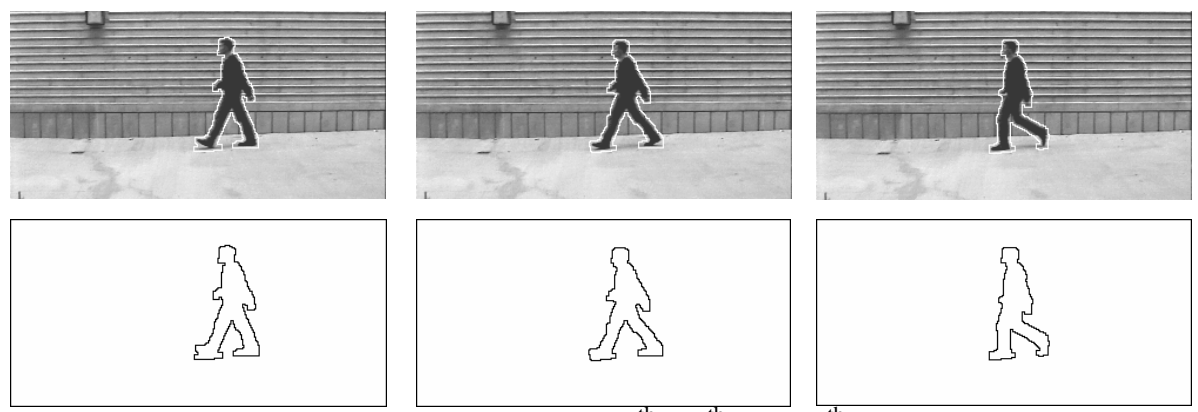

(b) the person \#4 $\left(33^{\text {th }}, 36^{\text {th }}\right.$ and $37^{\text {th }}$ frame $)$
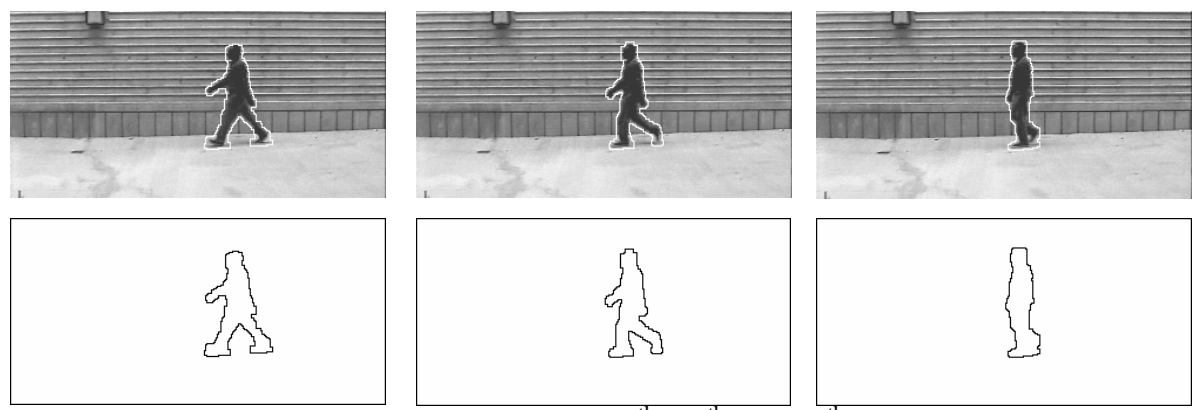

(c) the person $\# 6\left(33^{\text {th }}, 36^{\text {th }}\right.$ and $37^{\text {th }}$ frame $)$

Fig. 6. Results of human shape tracking on the other subjects: (a), (b) and (c) are a subject of UCSD database, respectively

To quantitatively assess of validity of the proposed human shape tracking method, we compare the results between the proposed method using mean-shift algorithm and the method only using GACMs without mean-shift algorithm. The comparison in term of convergence speed on a sequence is shown in Fig.5. In the proposed method, the curve evolving function is fast converged because initial curve is re-localized neared the human region using mean-shift algorithm. Due to it, the method only using ACM takes larger time to track the human shape than the proposed method.

Consequently, the proposed method yields accurate human shape tracking, so that it can be used effectively to extract the gait features for human identification. 


\section{Conclusions}

In this paper, we presented a human shape extraction and tracking method for gait recognition using geodesic active contour models (GACMs) combined with meanshift algorithm. Our system consists of two modules: the human region detection and the human shape tracking. In the human region detection module, the silhouette of a walking person is extracted by background subtraction and morphologic operation. Then human shapes are correctly obtained by the GAC with mean-shift algorithm. The main idea is very simple. Before evolving using level-set method, the initial curve in each frame is re-localized near the human region and is resized enough to include the target object. To evaluate the effectiveness of the proposed method, it was applied the common gait data, then the results showed that the proposed method is extracted and tracked efficiently accurate shape for gait recognition.

\section{Acknowledgments}

This work was supported by the Korea Research Foundation Grant (KRF-2006-331D00545).

\section{References}

1. Wang, L., Ning, H., Tan, T., Hu, W.: Automatic Gait Recognition Based on Statistical Shape Analysis. IEEE Transaction on Image Processing, 1120-1131 (2003)

2. Lee, L., Grimson, W.: Gait analysis for recognition and classification. In: Proceedings of the International Conference on Automatic Face and Gesture Recognition, pp. 155-162 (2002)

3. Collins, R., Cross, R., Shi, J.: Silhouette-based Human Identification from Body Shape and Gait. In: Proceedings of the International Conference on Face and Gesture Recognition, pp. 366-371 (2002)

4. Vega, I., Sarkar, S.: Statistical Motion Model1 Based on the Change of Feature Relationships: Human Gait-Based Recognition. IEEE Transactions on Pattern Analysis and Machine Intelligence, 1323-1328 (2003)

5. Sarkar, S., Phillips, P.J., Liu, Z., Vega, I.R., Grother, P., Bowyer, K.: The HumnaID Gait Challenge Problem: Data Sets, Performance and Analysis. IEEE Transactions on Pattern Analysis and Machine Intelligence, 162-177 (2005)

6. Liu, L., Zhang, S., Zhang, Y., Ye, X.: Human Contour Extraction Using Level Set. In: Proceedings of the International Conference on Computer and Information Technology pp. 608-612 (2005)

7. Paragios, N., Deriche, R.: Geodesic Active Contours and Level Sets for the Detection and Tracking of Moving Objects. IEEE Transactions on Pattern Analysis and Machine Intelligence, 266-279 (2000)

8. Little, J., Boyd, J.: Recognizing People by Their Gait Description via Temporal Moments. Videre 1(2), 1-33 (1998)

9. Yang, Y., Levine, M.: The Background primal sketch: An approach for tracking moving objects. Mach. Vis. Applicant, 17-34 (1992) 
10. Kim, K.I., Jung, K., Kim, H.J.: Texture-Based Approach for Text Detection in Image Using Support Vector Machines and Continuously Adaptive Mean Shift Algorithm. IEEE Transactions on Pattern Analysis and Machine Intelligence, 1631-1639 (2003)

11. Jaffre, G., Crouzil, A.: Non-rigid Object Localization From Color Model Using Mean Shift. In: Proceedings of the International Conference on Image Processing, vol. 3, pp. 317-319 (2003)

12. Caselles, V., Kimmel, R., Sapiro, G.: Geodesic Active Contours. In: Proceedings of IEEE the International Conference on Computer Vision, pp. 694-699 (1995) 